\title{
Production and Detection of an Axion Dark Matter Echo
}

\author{
Ariel Arza and Pierre Sikivie \\ Department of Physics, University of Florida, Gainesville, Florida 32611, USA
}

(Received 4 February 2019; revised manuscript received 19 May 2019; published 27 September 2019)

\begin{abstract}
Electromagnetic radiation with angular frequency equal to half the axion mass stimulates the decay of cold dark matter axions and produces an echo, i.e., faint electromagnetic radiation traveling in the opposite direction. We propose to search for axion dark matter by sending out to space a powerful beam of microwave radiation and listening for its echo. We estimate the sensitivity of this technique in the isothermal and caustic ring models of the Milky Way halo and find it to be a promising approach to axion, or axionlike, dark matter detection.
\end{abstract}

DOI: 10.1103/PhysRevLett.123.131804

The identity of dark matter remains one of the central questions in science today [1]. One of the leading candidates is the QCD axion. This hypothetical particle was originally postulated as a solution [2] to the strong $C P$ problem of the standard model of particle physics, i.e., the puzzle why the strong interactions conserve $P$ and $C P$. The properties of the QCD axion are given almost entirely in terms of a single parameter $f_{a}$, called the axion decay constant. In particular, the mass of the axion

$$
m=0.6 \times 10^{-5} \mathrm{eV}\left(\frac{10^{12} \mathrm{GeV}}{f_{a}}\right),
$$

and its electromagnetic coupling

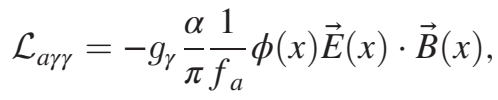

where $\phi(x)$ is the axion field and $g_{\gamma}$ is a model-dependent dimensionless coupling that is generically of order one. In the Kim-Shifman-Vainshtein-Zakharov (KSVZ) model [3], $g_{\gamma}=-0.97$. In the Dine-Fischler-Srednicki-Zhitnitskii (DFSZ) model [4], and in all grand-unified axion models, $g_{\gamma}=0.36$. Early laboratory limits and stellar evolution constraints require $f_{a}>10^{9} \mathrm{GeV}$ [5], in which case the axion is so extremely weakly coupled that it was once dubbed "invisible." However, cosmology came to the rescue. Axions are overproduced during the QCD phase transition in the simplest cosmological scenarios unless $f_{a} \lesssim 10^{12} \mathrm{GeV}$ [6]. The precise limit depends on whether inflation occurs before or after the phase transition during

Published by the American Physical Society under the terms of the Creative Commons Attribution 4.0 International license. Further distribution of this work must maintain attribution to the author(s) and the published article's title, journal citation, and DOI. Funded by SCOAP ${ }^{3}$. which Peccei-Quinn symmetry is spontaneously broken and other considerations, such axion production by topological defects, the precise temperature dependence of the axion mass, and the amount of entropy production associated with the QCD phase transition. In any case, the axions produced during the QCD phase transition are a form of cold dark matter [7] and therefore a candidate for the constituent particle of galactic halos. The topic is reviewed in Refs. [8].

Axions and axionlike particles (ALPs) are by-products of variously motivated proposals for physics beyond the standard model, including its supersymmetric extensions [9] and string theory [10]. ALPs [11] are light pseudoscalar particles like QCD axions, but without the definite relationship between mass and coupling implied by Eqs. (1) and (2). Several methods to test experimentally the axion hypothesis have been proposed and some have produced useful limits. For dark matter axion searches, the different approaches include the cavity technique $[12,13]$, wire [14] and dielectric plate [15] detectors, magnetic resonance methods [16,17], the $L C$ circuit approach [18], and atomic transitions [19]. Solar axions are searched for by their conversion to $\mathrm{x}$ rays in a laboratory magnetic field $[12,20]$ and in crystals [21] and through the axioelectric effect [22]. "Shining light through wall" experiments attempt to convert photons to axions on one side of a wall followed by backconversion on the other side [23]. Axion induced effects in atoms, molecules, and nuclei are discussed in Ref. [24].

The purpose of our Letter is to propose a new approach to axion dark matter detection. The effect we exploit is the stimulated decay of cold dark matter axions by a powerful beam of microwave radiation. References [25] discuss the stimulated decay of axion dark matter in astrophysical contexts. We first describe the effect in the rest frame of a perfectly cold axion fluid and then generalize to the case where the observer is moving with respect to the axion fluid and to the case where the axion fluid has velocity dispersion. 
Let $\vec{A}_{0}(\vec{x}, t)$ be the vector potential of the initial outgoing radiation. In the presence of axions, $\vec{A}_{0}$ is itself a source of electromagnetic radiation $\vec{A}_{1}(\vec{x}, t)$. Since axions are very weakly coupled, we have in radiation gauge $(\vec{\nabla} \cdot \vec{A}=0)$

$$
\left(\partial_{t}^{2}-\nabla^{2}\right) \vec{A}_{1}=-g\left(\vec{\nabla} \times \vec{A}_{0}\right) \partial_{t} \phi,
$$

where $\phi(t)=A \sin (m t)$ is the axion field, and $g \equiv$ $g_{\gamma}(\alpha / \pi)\left(1 / f_{a}\right)$ is the overall coupling that appears in Eq. (2). The axion density is $\rho=\frac{1}{2} A^{2} m^{2}$. Let the outgoing radiation $\vec{A}_{0}$ be stationary, linearly polarized, and with angular frequencies $\omega$ at and near $m / 2$. The retarded $\vec{A}_{1}$ is in that case identical to $\vec{A}_{0}$ except that (1) it flows exactly backwards because, up to a constant factor, its spatial Fourier transform is the same as that of $\vec{A}_{0}$, whereas its angular frequency is opposite, (2) it is reduced relative to $\vec{A}_{0}$ by a time-dependent factor proportional to $g A$, and (3) it is linearly polarized at a $90^{\circ}$ angle relative to $\vec{A}_{0}$ (see Supplemental Material [26]). If $\vec{A}_{0}$ is circularly polarized, $\vec{A}_{1}$ has the same circular polarization as $\vec{A}_{0}$. We call $\vec{A}_{1}$ the echo wave.

The power in the echo wave is [26]

$$
P_{1}=\frac{1}{16} g^{2} \rho \frac{d P_{0}}{d \nu} t
$$

where $d P_{0} / d \nu$ is the spectral density of the outgoing wave at angular frequency $\omega=2 \pi \nu=m / 2$, and $t$ is the time since the outgoing wave was first established. If the outgoing wave is emitted as a parallel beam of finite cross section, it will spread as a result of its transverse wave vector components. The echo wave retraces the outgoing wave backward in time, returning to the location of emission of the outgoing wave with the latter's original transverse size. If the outgoing power $P_{0}$ is turned on for a time $t$ and then turned off, the echo power $P_{1}$ given by Eq. (4) lasts forever in the future under the assumption that the perfectly cold axion fluid has infinite spatial extent.

Next, let us consider the case where the perfectly cold axion fluid is moving with velocity $\vec{v}$ with respect to the outgoing power source. Nothing changes in the axion fluid rest frame compared to the above discussion except that each increment $d E_{0}=P_{0} d t$ of outgoing energy is emitted from a different location. The incremental echo power $d P_{1}$, given by the rhs of Eq. (4) with $t$ replaced by $d t$, returns forever to the location in the axion fluid rest frame from which the increment $d E_{0}$ of outgoing energy was emitted. In the frame of its source, the frequency at which the outgoing power stimulates axion decay is $(c=1)$

$$
\omega_{0}=\frac{m}{2}(1+\vec{v} \cdot \hat{k})+\mathcal{O}\left(v^{2}\right)
$$

where $\hat{k}$ is the unit vector in the direction of the outgoing power. The frequency of the echo is

$$
\omega_{-}=\frac{m}{2}(1-\vec{v} \cdot \hat{k})+\mathcal{O}\left(v^{2}\right),
$$

and its direction is $-\hat{k}+2 \vec{v}_{\perp}+\mathcal{O}\left(v^{2}\right)$, where $\vec{v}_{\perp}$ is the component of $\vec{v}$ perpendicular to $\hat{k}$. The echo of power emitted a time $t_{e}$ ago arrives displaced from the point of emission by $\vec{d}=\vec{v}_{\perp} t_{e}$. To detect as much echo power as possible at or near the place of emission of the outgoing power, the observer wants $\vec{v}_{\perp}$ as small as possible, i.e., $\hat{k}$ parallel or antiparallel to $\vec{v}$.

If the axion fluid has velocity dispersion, its density can be viewed as an integral over cold flows

$$
\rho=\int d^{3} v \frac{d^{3} \rho}{d v^{3}}(\vec{v})
$$

Everything said before holds true for each infinitesimal cold flow increment. The echo frequency has a spread $\delta \omega_{-}=(m / 2) \delta v_{\|}$, where $\delta v_{\|}$is the spread of axion velocities in the $\hat{k}$ direction. The echo of power emitted a time $t_{e}$ ago is spread over a transverse size $\delta \vec{d}=\delta \vec{v}_{\perp} t_{e}$, where $\delta \vec{v}_{\perp}$ is the spread of axion velocities perpendicular to $\hat{k}$.

It is clear from the above that the amount of echo power that the observer may easily collect depends sharply on the velocity distribution of axion dark matter on Earth as well as its total local density. We will consider two contrasting models of the Milky Way halo, the isothermal model [27] and the caustic ring model [28]. In the isothermal model, the local dark matter has density $300 \mathrm{MeV} / \mathrm{cm}^{3}$ and velocity dispersion $270 \mathrm{~km} / \mathrm{s}$. In the caustic ring model, the local dark matter velocity distribution is dominated by a single flow, called the big flow, because of our proximity to the fifth caustic ring of dark matter in the Milky Way halo. An upper limit of order $70 \mathrm{~m} / \mathrm{s}$ on the velocity dispersion of the big flow has been derived [29]. The direction of the big flow can be obtained from triangular features in the Infrared Astronomical Satellite (IRAS) and Global Astrometric Interferometer for Astrophysics (GAIA) maps of the Galactic plane [30] with a precision of order $0.01 \mathrm{rad}$. The density of the big flow is poorly constrained because it depends sharply on our distance and position relative to the midplane cusp of the nearby caustic ring. It is $1 \mathrm{GeV} / \mathrm{cm}^{3}$ [28] at least, but may be as large as $10 \mathrm{GeV} / \mathrm{cm}^{3}$ or even higher [30]. Such high densities pertain only to a small region within $10 \mathrm{pc}$ or so of the aforementioned cusp and are consistent with measurements of the Galactic rotation curve and of the vertical motions of stars in the solar neighborhood.

Let us first consider the case where the local axion dark matter density is dominated by a single cold flow, as in the caustic ring model. As was discussed above, the largest 
amount of echo power is available for detection near the source of outgoing power when the outgoing power has direction $\hat{k}$ parallel or antiparallel to the velocity vector $\vec{v}$ of the axion fluid with respect to the observer. That velocity vector is a sum

$$
\vec{v}(t)=\vec{v}_{a}-\vec{v}_{\mathrm{LSR}}-\vec{v}_{\odot}-\vec{v}_{\otimes}(t),
$$

where $\vec{v}_{a}$ is the velocity of the axion fluid with respect to a nonrotating coordinate system attached to the Milky Way Galaxy, $\vec{v}_{\text {LSR }}$ is the velocity of the local standard of rest (LSR) in that same coordinate system, $\vec{v}_{\odot}$ is the velocity of the Sun with respect to the LSR, and $\vec{v}_{\otimes}$ is the velocity of the observer with respect to the Sun as a result of the orbital and rotational motions of Earth. We are particularly interested in the extent to which the uncertainties in the several terms on the rhs of Eq. (8) affect our ability to minimize $\vec{v}_{\perp} \cdot \vec{v}_{\otimes}(t)$ is known with great precision. The components of $\vec{v}_{\odot}$ are known with a precision of order $3 \mathrm{~km} / \mathrm{s} . \vec{v}_{\mathrm{LSR}}$ is in the direction of Galactic rotation by definition. Its magnitude (often quoted to be $220 \mathrm{~km} / \mathrm{s}$ ) is known with an uncertainty of order $20 \mathrm{~km} / \mathrm{s}$. The magnitude of $\vec{v}_{a}$ for the big flow is approximately $520 \mathrm{~km} / \mathrm{s}$ [28]. Its direction is fixed by the positions of the IRAS and GAIA triangles on the sky with a precision of order $0.01 \mathrm{rad}$ [30]. So we expect that it is not possible to reduce $\vec{v}_{\perp}$ to less than of order $5 \mathrm{~km} / \mathrm{s}$, the nominal value we use below. Because the big flow is almost in the direction of Galactic rotation (within approximately $12^{\circ}$ ), the uncertainty on the magnitude of $\vec{v}_{\text {LSR }}$ is less important.

Consider a dish (e.g., a radiotelescope) of radius $R$ collecting echo power at or near the location of the outgoing power source. Because the echo from outgoing power emitted a time $t_{e}$ ago is displaced by $\vec{d}=\vec{v}_{\perp} t_{e}$, the amount of echo power collected by the dish is

$$
P_{c}=\frac{1}{16} g^{2} \rho \frac{d P_{0}}{d \nu} C \frac{R}{\left|\vec{v}_{\perp}\right|},
$$

where $C$ is a number of order one that depends on the configuration of the source relative to the receiver dish

$$
C=\frac{\left|\vec{v}_{\perp}\right|}{2 R P_{0}} \int d t \int_{S_{0}} d^{2} x I_{0}(\vec{x}) \Theta_{c}\left(\vec{x}+\vec{v}_{\perp} t\right) .
$$

Here $S_{0}$ is the surface from which the outgoing power is emitted, $I_{0}(\vec{x})$ is the outgoing power per unit surface, and $\Theta_{c}(\vec{x})$ is a function that equals one if $\vec{x}$ belongs to the receiver dish area and zero otherwise. For example, $C=0.5$ if the outgoing power is emitted from the center of the receiver dish, whereas $C=0.424$ if the outgoing power is emitted uniformly from the area of the receiver dish. However, neither of these configurations is likely to be optimal. It is probably better to place several source dishes around the receiver dish. $C$ can be straightforwardly calculated for each configuration.

Let us assume that a pulse of outgoing power $P_{0}$, with frequency $\nu_{0}$ and uniform spectral density $d P_{0} / d \nu=$ $P_{0} / \Delta \nu_{0}$ over bandwidth $\Delta \nu_{0}$, is emitted during a time $t_{m}$. Provided that

$$
t_{m}>\frac{R}{2\left|\vec{v}_{\perp}\right|}=0.5 \times 10^{-2} \mathrm{~s} \frac{R}{50 \mathrm{~m}} \frac{5 \mathrm{~km} / \mathrm{s}}{\left|\vec{v}_{\perp}\right|},
$$

the echo power

$$
\begin{aligned}
P_{c}= & 2.33 \times 10^{-31} P_{0}\left(\frac{10 \mathrm{kHz}}{\Delta \nu_{0}}\right)\left(\frac{g_{\gamma}}{0.36}\right)^{2}\left(\frac{10^{12} \mathrm{GeV}}{f_{a}}\right)^{2} \\
& \times\left(\frac{\rho}{\mathrm{GeV} / \mathrm{cm}^{3}}\right)\left(\frac{C}{0.30}\right)\left(\frac{R}{50 \mathrm{~m}}\right)\left(\frac{5 \mathrm{~km} / \mathrm{s}}{\left|\vec{v}_{\perp}\right|}\right)
\end{aligned}
$$

is received over the same time interval $t_{m}$. Since the magnitude of the velocity of the big flow relative to us $v \simeq 520-220=300 \mathrm{~km} / \mathrm{s}$, the frequency of the echo power is red- or blueshifted from $\nu_{0}$ by $\Delta \nu \simeq 2 \times 10^{-3} \nu_{0}$. The echo power has bandwidth $B=2 \delta v \nu<5 \times 10^{-7} \nu$ since the velocity dispersion of the big flow is less than $70 \mathrm{~m} / \mathrm{s}$ [29]. The frequency range of interest is approximately $30 \mathrm{MHz}-30 \mathrm{GHz}$ because Earth's atmosphere is mostly transparent at those frequencies. It corresponds to the mass range $2.5 \times 10^{-7}<m<2.5 \times 10^{-4} \mathrm{eV}$, which happens to be prime hunting ground for QCD axions.

The cosmic microwave background and radio emission by astrophysical sources are irreducible sources of noise. In addition there is instrumental noise. The total noise temperature depends on frequency, on the location of the telescope, and on the direction of observation. As an example, we may consider the system noise temperature of the Green Bank Telescope [31]: approximately $20 \mathrm{~K}$ from 1 to $8 \mathrm{GHz}$, approximately linearly rising from $20 \mathrm{~K}$ at $8 \mathrm{GHz}$ to $40 \mathrm{~K}$ at $30 \mathrm{GHz}$, and exponentially rising towards low frequencies from $20 \mathrm{~K}$ at $1 \mathrm{GHz}$ to $100 \mathrm{~K}$ at $300 \mathrm{MHz}$. The rise at low frequencies is due to galactic emission and is strongly direction dependent. A typical value is $100 \mathrm{~K}$ at $300 \mathrm{MHz}$. The rise at high frequencies is due to atomic and molecular transitions in the atmosphere. It can be mitigated by placing the telescope at a high elevation. We use below a nominal system noise temperature of $20 \mathrm{~K}$ at all frequencies for the purpose of stating the results of our sensitivity calculations.

The signal-to-noise ratio with which the echo power is detected when $\omega_{0}$ falls within the angular frequency range of the emitted power is given by Dicke's radiometer equation

$$
s / n=\frac{P_{c}}{T_{n}} \sqrt{\frac{t_{m}}{B}} .
$$

Combining Eqs. (12) and (13) and setting $B=5 \times 10^{-7} \nu$, the total outgoing energy per logarithmic frequency interval 


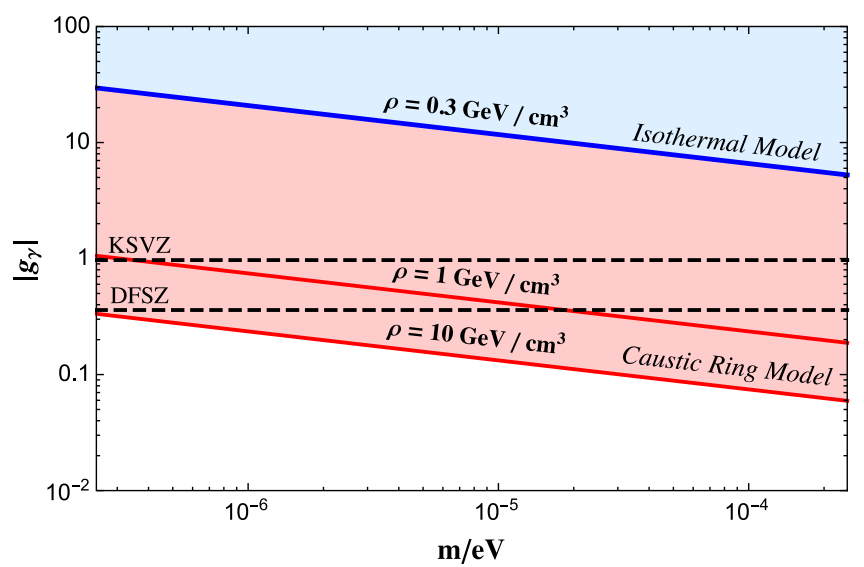

FIG. 1. Sensitivity to $\left|g_{\gamma}\right|$ as function of mass of an axion echo search consuming $10 \mathrm{MW}$ yr of outgoing energy for each factor 2 in axion mass range covered, in the caustic ring model with big flow densities $\rho=1$ and $10 \mathrm{GeV} / \mathrm{cm}^{3}$, and in the isothermal model with density $\rho=0.30 \mathrm{GeV} / \mathrm{cm}^{3}$, assuming all other experimental parameters have the nominal values shown in Eqs. (14) and (16).

necessary to detect the axion echo with a given signal-tonoise ratio is found to be

$$
\begin{aligned}
\left.\frac{d E_{0}}{d \ln \nu}\right|_{\mathrm{BF}}= & 7.2 \mathrm{MW} y r\left(\frac{s / n}{5}\right)\left(\frac{10 \mathrm{GHz}}{\nu}\right)^{1 / 2}\left(\frac{0.36}{g_{\gamma}}\right)^{2} \\
& \times\left(\frac{T_{n}}{20 \mathrm{~K}}\right)\left(\frac{\mathrm{GeV} / \mathrm{cm}^{3}}{\rho}\right)\left(\frac{0.30}{C}\right) \\
& \times\left(\frac{t_{m}}{10^{-2} \mathrm{~s}}\right)^{1 / 2}\left(\frac{50 \mathrm{~m}}{R}\right)\left(\frac{\left|v_{\perp}\right|}{5 \mathrm{~km} / \mathrm{s}}\right) .
\end{aligned}
$$

We used Eq. (1) and $m=4 \pi \nu$. Figure 1 shows the sensitivity to $g_{\gamma}$ of an axion echo search that consumes $10 \mathrm{MWyr}$ of outgoing energy for each octave (factor of 2) in axion mass covered, for $\rho=1$ and $10 \mathrm{GeV} / \mathrm{cm}^{3}$ and the nominal values of all other experimental parameters used in Eq. (14).

In the isothermal model, $\vec{v}_{a}=0$ on average. In a nonrotating galactic reference frame, the velocity distribution is Gaussian with dispersion $\sqrt{3} \sigma \equiv \sqrt{\langle\vec{v} \cdot \vec{v}\rangle} \simeq 270 \mathrm{~km} / \mathrm{s}$. In the LSR, the axion fluid moves with speed $220 \mathrm{~km} / \mathrm{s}$ in the direction opposite to that of galactic rotation. Assuming the direction $\hat{k}$ of the outgoing power is parallel (antiparallel) to the direction of galactic rotation, the echo power is blueshifted (redshifted) in frequency by a fractional amount whose average is $\langle\Delta \nu / \nu\rangle \simeq 440 \mathrm{~km} / \mathrm{s}=1.5 \times 10^{-3}$ and whose rms deviation is $\delta \nu / \nu=2 \sigma \simeq 1.04 \times 10^{-3}$. The echo from outgoing energy that was emitted a time $t_{e}$ ago is spread in space over a fuzzy circular region whose radius is Gaussian distributed with average value $\sigma t_{e}$. Equation (9) holds with $1 /\left|\vec{v}_{\perp}\right|$ replaced by

$$
\left\langle\frac{1}{\left|\vec{v}_{\perp}\right|}\right\rangle=\sqrt{\frac{\pi}{2}} \frac{1}{\sigma}=\frac{1}{124 \mathrm{~km} / \mathrm{s}} .
$$

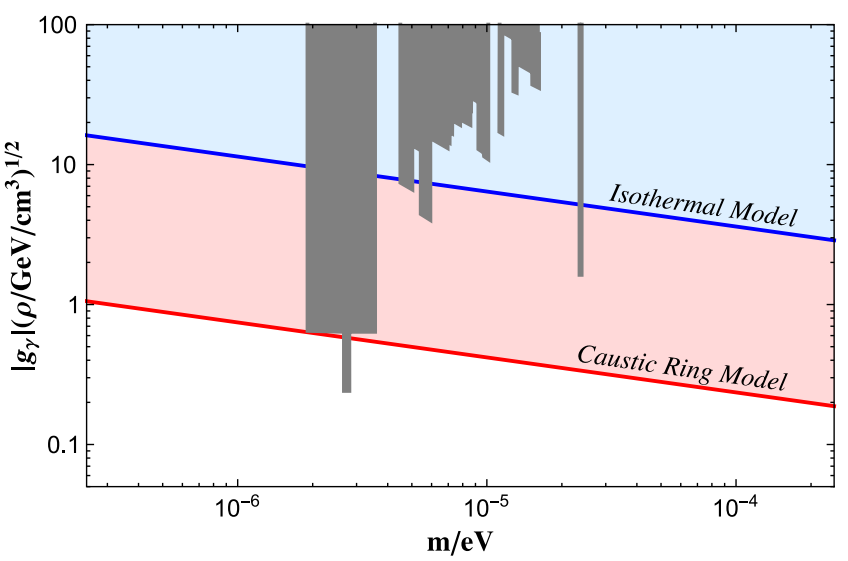

FIG. 2. Sensitivity to $\left|g_{\gamma}\right| \sqrt{\rho}$ as a function of mass of an axion echo search consuming $10 \mathrm{MW}$ yr of outgoing energy for each factor 2 in axion mass range searched, in the caustic ring model and in the isothermal model, assuming the nominal values of the experimental parameters shown in Eqs. (14) and (16). The gray shaded areas are ruled out by searches using the cavity method.

In view of Eq. (11) we now require $t_{m}>2 \times 10^{-4} \mathrm{~s}(R / 50 \mathrm{~m})$. Using Eq. (13) with $B=4 \sigma \nu=2.1 \times 10^{-3} \nu$ and setting $\rho=0.3 \mathrm{GeV} / \mathrm{cm}^{3}$, we find

$$
\begin{aligned}
\left.\frac{d E_{0}}{d \ln \nu}\right|_{\text {iso }}= & 5.3 \mathrm{GWyr}\left(\frac{s / n}{5}\right)\left(\frac{10 \mathrm{GHz}}{\nu}\right)^{1 / 2}\left(\frac{0.36}{g_{\gamma}}\right)^{2} \\
& \times\left(\frac{T_{n}}{20 \mathrm{~K}}\right)\left(\frac{0.30}{C}\right)\left(\frac{t_{m}}{2 \times 10^{-4} \mathrm{~s}}\right)^{1 / 2}\left(\frac{50 \mathrm{~m}}{R}\right) .
\end{aligned}
$$

The sensitivity to $g_{\gamma}$ in the isothermal model is shown in Fig. 1 as well.

The echo method appears an attractive approach to axion dark matter detection because it uses relatively old technology and because it is applicable over a wide range of axion masses. The method works better in the caustic ring model than in the isothermal model for three reasons: (1) the density is higher, (2) the echo has less spread in frequency, and (3) the echo has less spread in physical space. Higher density helps the cavity method equally. Figure 2 shows the sensitivity of the echo method to $\left|g_{\gamma}\right| \sqrt{\rho}$, as well as the published limits obtained by searches using the cavity method. The small spread in frequency of a signal from a cold flow, such as the big flow of the caustic ring model, also somewhat helps the cavity method. Reference [32] describes a high resolution analysis of Axion Dark Matter eXperiment (ADMX) data and shows that an improvement by a factor of order 2 in $g_{\gamma} \sqrt{\rho}$ is obtained in case of a cold flow of velocity dispersion less than $10 \mathrm{~m} / \mathrm{s}$. The improvements in sensitivity of the cavity method in case of cold flows are not included in the published bounds shown in Fig. 2. 
We thank Richard Bradley, Fritz Caspers, Guido Mueller, Neil Sullivan, David Tanner, Qiaoli Yang, and Konstantin Zioutas for useful discussions. This research was supported in part by the U.S. Department of Energy under Award No. DE-SC0010296, by the Chilean Commission on Research, Science and Technology (CONICYT) under Grant No. 78180100 (Becas Chile, Postdoctorado), and by the Heising-Simons Foundation under Grant No. 2015-109.

[1] Reviews include Particle Dark Matter, edited by G. Bertone (Cambridge University Press, Cambridge, England, 2010); E. W. Kolb and M. Turner, The Early Universe (AddisonWesley, Reading, MA, 1990).

[2] R. D. Peccei and H. R. Quinn, Phys. Rev. Lett. 38, 1440 (1977); Phys. Rev. D 16, 1791 (1977); S. Weinberg, Phys. Rev. Lett. 40, 223 (1978); F. Wilczek, Phys. Rev. Lett. 40, 279 (1978).

[3] J. Kim, Phys. Rev. Lett. 43, 103 (1979); M. A. Shifman, A. I. Vainshtein, and V. I. Zakharov, Nucl. Phys. B166, 493 (1980).

[4] M. Dine, W. Fischler, and M. Srednicki, Phys. Lett. B 104B, 199 (1981); A. Zhitnitskii, Sov. J. Nucl. 31, 260 (1980).

[5] G. Raffelt, Lect. Notes Phys. D 79, 51 (2008).

[6] J. Preskill, M. Wise, and F. Wilczek, Phys. Lett. B 120B, 127 (1983); L. Abbott and P. Sikivie, Phys. Lett. B 120B, 133 (1983); M. Dine and W. Fischler, Phys. Lett. 120B, 137 (1983).

[7] J. Ipser and P. Sikivie, Phys. Rev. Lett. 50, 925 (1983).

[8] P. Sikivie, Lect. Notes Phys. 741, 19 (2008).D. J. E. Marsh, Phys. Rep. 643, 1 (2016).

[9] H. Baer, V. Barger, and D. Sengupta, Phys. Lett. B 790, 58 (2019), and references therein.

[10] P. Svrcek and E. Witten, J. High Energy Phys. 06 (2006) 051.

[11] P. Arias, D. Cadamuro, M. Goodsell, J. Jaeckel, J. Redondo, and A. Ringwald, J. Cosmol. Astropart. Phys. 06 (2012) 013, and references therein.

[12] P. Sikivie, Phys. Rev. Lett. 51, 1415 (1983); Phys. Rev. D 32, 2988 (1985).

[13] S. J. Asztalos et al., Phys. Rev. Lett. 104, 041301 (2010); N. Du et al., Phys. Rev. Lett. 120, 151301 (2018); B. M. Brubaker et al., Phys. Rev. Lett. 118, 061302 (2017); S. Youn, Int. J. Mod. Phys. Conf. Ser. 43, 1660193 (2016); B. T. McAllister, G. Flower, E. N. Ivanov, M. Goryachev, J. Bourhill, and M. E. Tobar, Phys. Dark Universe 18, 67 (2017).

[14] P. Sikivie, D. B. Tanner, and Y. Wang, Phys. Rev. D 50, 4744 (1994); G. Rybka and A. Wagner, Phys. Rev. D 91, 011701(R) (2015).

[15] A. Caldwell, G. Dvali, B. Majorovits, A. Millar, G. Raffelt, J. Redondo, O. Reimann, F. Simon, and F. Steffen, Phys. Rev. Lett. 118, 091801 (2017).

[16] P. W. Graham and S. Rajendran, Phys. Rev. D 88, 035023 (2013); D. Budker, P. W. Graham, M. Ledbetter, S. Rajendran, and A. O. Sushkov, Phys. Rev. X 4, 021030 (2014);
P. Graham, D. E. Kaplan, J. Mardon, S. Rajendran, W. A. Terrano, L. Trahms, and T. Wilkason, Phys. Rev. D 97, 055006 (2018).

[17] R. Barbieri, C. Braggio, G. Carugno, C. S. Gallo, A. Lombardi, A. Ortolan, R. Pengo, G. Ruoso, and C. C. Speake, Phys. Dark Universe 15, 135 (2017).

[18] P. Sikivie, N. Sullivan, and D. B. Tanner, Phys. Rev. Lett. 112, 131301 (2014); Y. Kahn, B. R. Safdi, and J. Thaler, Phys. Rev. Lett. 117, 141801 (2016); B. T. McAllister, S. R. Parker, and M.E. Tobar, Phys. Rev. D 94, 042001 (2016); N. Crisosto, G. Rybka, and K. van Bibber, Springer Proc. Phys. 211, 127 (2018); J. L. Ouellet et al., Phys. Rev. Lett. 122, 121802 (2019).

[19] P. Sikivie, Phys. Rev. Lett. 113, 201301 (2014); C. Braggio et al., Sci. Rep. 7, 15168 (2017); V. V. Flambaum, H. B. Tran Tan, I. B. Samsonov, Y. V. Stadnik, and D. Budker, Int. J. Mod. Phys. A 33, 1844030 (2018).

[20] Y. Inoue, Y. Akimoto, R. Ohta, T. Mizumoto, A. Yamamoto, and M. Minowa, Phys. Lett. B 668, 93 (2008); V. Anastassopoulos et al., Nat. Phys. 13, 584 (2017); E. Armengaud et al., J. Instrum. 9, T05002 (2014).

[21] F. Avignone et al., Phys. Rev. Lett. 81, 5068 (1998).

[22] S. Dimopoulos, G. D. Starkman, and B. W. Lynn, Phys. Lett. 168B, 145 (1986); D. S. Akerib et al., Phys. Rev. Lett. 118, 261301 (2017), and references therein.

[23] K. van Bibber, N. R. Dagdeviren, S. E. Koonin, A. K. Kerman, and H. N. Nelson, Phys. Rev. Lett. 59, 759 (1987); K. Ehret et al., Phys. Lett. B 689, 149 (2010), and references therein; R. Ballou et al., Phys. Rev. D 92, 092002 (2015); F. Hoogeveen and T. Ziegenhagen, Nucl. Phys. B358, 3 (1991); G. Mueller, P. Sikivie, D. Tanner, and K. van Bibber, Phys. Rev. D 80, 072004 (2009).

[24] Y. V. Stadnik and V. V. Flambaum, Phys. Rev. D 89, 043522 (2014).

[25] I. I. Tkachev, JETP Lett. 101, 1 (2015); A. Arza, Eur. Phys. J. C 79, 250 (2019); A. Caputo, M. Regis, M. Taoso, and S. J. Witte, J. Cosmol. Astropart. Phys. 03 (2019) 027; A. Caputo, C. P. Garay, and S. J. Witte, Phys. Rev. D 98, 083024 (2018); 99, 089901(E) (2019).

[26] See Supplemental Material at http://link.aps.org/ supplemental/10.1103/PhysRevLett.123.131804 for a derivation of Eq. (4) and additional figure.

[27] M. S. Turner, Phys. Rev. D 33, 889 (1986).

[28] L. D. Duffy and P. Sikivie, Phys. Rev. D 78, 063508 (2008).

[29] N. Banik and P. Sikivie, Phys. Rev. D 93, 103509 (2016).

[30] S. Chakrabarty, A. Gonzales, Y. Han, and P. Sikivie (to be published).

[31] https://science.nrao.edu/facilities/gbt/proposing/GBTpg.pdf.

[32] L. Duffy, P. Sikivie, D. B. Tanner, S. Asztalos, C. Hagmann, D. Kinion, L. J. Rosenberg, K. van Bibber, D. Yu, and R. F. Bradley, Phys. Rev. Lett. 95, 091304 (2005); Phys. Rev. D 74, 012006 (2006). 